\title{
Numerical Solution of Nonlinear Fredholm Integrodifferential Equations of Fractional Order by Using Hybrid Functions and the Collocation Method
}

\author{
Jianhua Hou, Beibo Qin, and Changqing Yang \\ Department of Science, Huaihai Institute of Technology, Lianyungang, Jiangsu 222005, China
}

Correspondence should be addressed to Changqing Yang, cdruby@126.com

Received 24 November 2011; Revised 4 February 2012; Accepted 12 February 2012

Academic Editor: Ch Tsitouras

Copyright (c) 2012 Jianhua Hou et al. This is an open access article distributed under the Creative Commons Attribution License, which permits unrestricted use, distribution, and reproduction in any medium, provided the original work is properly cited.

\begin{abstract}
A numerical method to solve nonlinear Fredholm integral equations of second kind is presented in this work. The method is based upon hybrid function approximate. The properties of hybrid of block-pulse functions and Taylor series are presented and are utilized to reduce the computation of nonlinear Fredholm integral equations to a system of algebraic equations. Some numerical examples are selected to illustrate the effectiveness and simplicity of the method.
\end{abstract}

\section{Introduction}

Over the last years, the fractional calculus has been used increasingly in different areas of applied science. This tendency could be explained by the deduction of knowledge models which describe real physical phenomena. In fact, the fractional derivative has been proved reliable to emphasize the long memory character in some physical domains especially with the diffusion principle. For example, the nonlinear oscillation of earthquake can be modeled with fractional derivatives, and the fluid-dynamic traffic model with fractional derivatives can eliminate the deficiency arising from the assumption of continuum traffic flow [1]. In the fields of physics and chemistry, fractional derivatives and integrals are presently associated with the application of fractals in the modeling of electrochemical reactions, irreversibility and electromagnetism [2], heat conduction in materials with memory, and radiation problems. Many mathematical formulations of mentioned phenomena contain nonlinear integrodifferential equations with fractional order. Nonlinear phenomena are also of fundamental importance in various fields of science and engineering. The nonlinear 
models of real-life problems are still difficult to be solved either numerically or theoretically. There has recently been much attention devoted to the search for better and more efficient solution methods for determining a solution, approximate or exact, analytical or numerical, to nonlinear models $[3,4]$.

In this paper, we study the numerical solution of a nonlinear fractional integro-differential equation of the second kind:

$$
D^{\alpha} f(x)-\lambda \int_{0}^{1} k(x, t)[f(t)]^{q} d t=g(x), \quad q>1,
$$

with the initial condition

$$
f^{(i)}(0)=\delta_{i}, \quad i=0,1, \ldots, r-1, r-1<\alpha \leq r, r \in N,
$$

by hybrid of block-pulse functions and Taylor series. Here, $g \in L^{2}([0,1)), k \in L^{2}\left([0,1)^{2}\right)$ is known functions, and $f(x)$ is unknown function. $D^{\alpha}$ is the Caputo fractional differentiation operator and $q$ is a positive integer.

During the last decades, several methods have been used to solve fractional differential equations, fractional partial differential equations, fractional integro-differential equations and dynamic systems containing fractional derivatives, such as Adomian's decomposition method [5-9], He's variational iteration method [10-12], homotopy perturbation method [13, 14], homotopy analysis method [15], collocation method [16], Galerkin method [17], and other methods [18-20].

\section{Basic Definitions}

We give some basic definitions and properties of the fractional calculus theory which are used further in this paper.

Definition 2.1. The Riemann-Liouville fractional integral operator of order $\alpha \geq 0$ is defined as [21]

$$
\begin{aligned}
J^{\alpha} f(x) & =\frac{1}{\Gamma(\alpha)} \int_{0}^{x}(x-t)^{\alpha-1} f(t) d t, \quad \alpha>0, x>0, \\
J^{0} f(x) & =f(x) .
\end{aligned}
$$

It has the following properties

$$
J^{\alpha} x^{\gamma}=\frac{\Gamma(\gamma+1)}{\Gamma(\alpha+\gamma+1)} x^{\alpha+\gamma}, \quad \gamma>-1
$$

Definition 2.2. The Caputo definition of fractional derivative operator is given by

$$
D^{\alpha} f(x)=J^{m-\alpha} D^{m} f(x)=\frac{1}{\Gamma(m-\alpha)} \int_{0}^{x}(x-t)^{m-\alpha-1} f^{(m)}(t) d t
$$


where $m-1<\alpha \leq m, m \in N, x>0$. It has the following two basic properties

$$
\begin{gathered}
D^{\alpha} J^{\alpha} f(x)=f(x), \\
J^{\alpha} D^{\alpha} f(x)=f(x)-\sum_{k=0}^{m-1} f^{(k)}\left(0^{+}\right) \frac{x^{k}}{k !}, \quad x>0 .
\end{gathered}
$$

\section{Properties of Hybrid Functions}

\subsection{Hybrid Functions of Block-Pulse and Taylor Polynomials}

Hybrid functions $h_{n m}(x), n=1,2, \ldots, N, m=0,1,2, \ldots, M-1$, are defined on the interval $[0,1)$ as

$$
h_{n m}(x)= \begin{cases}T_{m}(N x-(n-1)), & x \in\left[\frac{n-1}{N}, \frac{n}{N}\right) \\ 0, & \text { otherwise }\end{cases}
$$

where $n$ and $m$ are the orders of block-pulse functions and Taylor polynomials, respectively and $T_{m}(x)=x^{m}$.

\subsection{Function Approximation}

A function $y(x) \in L^{2}[0,1)$ may be expanded as

$$
y(x)=\sum_{n=1}^{\infty} \sum_{m=0}^{\infty} c_{n m} h_{n m}(x)
$$

where

$$
c_{n m}=\left.\frac{1}{N^{m} m !}\left(\frac{d^{m} y(x)}{d x^{m}}\right)\right|_{x=((n-1) / N)} .
$$

If $y(x)$ in (3.2) is truncated, then (3.2) can be written as

$$
y(x)=\sum_{n=1}^{N} \sum_{m=0}^{M-1} c_{n m} h_{n m}(x) \approx C^{T} H(x)=H^{T}(x) C,
$$

where

$$
C=\left[c_{10}, c_{11}, \ldots, c_{1 M-1}, c_{20}, \ldots, c_{2 M-1}, \ldots c_{N 0}, \ldots, c_{N M-1}\right]^{T},
$$

$$
H(x)=\left[h_{10}(x), h_{11}(x), \ldots, h_{1 M-1}(x), h_{20}(x), \ldots, h_{2 M-1}(x), \ldots h_{N 0}(x), \ldots, h_{N M-1}(x)\right]^{T}
$$


In (3.5) and (3.6), $c_{n m}, n=1,2, \ldots, N, m=0,1, \ldots, M-1$, are the coefficients expansions of the function $y(x)$ in the $n$th subinterval $[(n-1) / N, n / N]$ and $h_{n m}(x), n=1,2, \ldots, N, m=$ $0,1, \ldots, M-1$, are defined in (3.1).

\subsection{Operational Matrix of the Fractional Integration}

The integration of the vector $H(t)$ defined in (3.6) can be obtained as

$$
\int_{0}^{t} H(\tau) d \tau \approx P H(t)
$$

see, [22], where $P$ is the $M N \times M N$ operational matrix for integration.

Our purpose is to derive the hybrid functions operational matrix of the fractional integration. For this purpose, we consider an $m$-set of block pulse function as

$$
b_{i}(t)= \begin{cases}1, & \frac{i}{m} \leq t \leq \frac{(i+1)}{m} \\ 0, & \text { otherwise }\end{cases}
$$

where $m=M N, i=0,1,2, \ldots,(m-1)$.

The functions $b_{i}(t)$ are disjoint and orthogonal. That is,

$$
b_{i}(t) b_{j}(t)= \begin{cases}0, & i \neq j, \\ b_{i}(t), & i=j .\end{cases}
$$

From the orthogonality of property, it is possible to expand functions into their block pulse series.

Similarly, hybrid function may be expanded into an $m$-set of block pulse function as

$$
H(t)=\Phi B(t)
$$

where $B(t)=\left[b_{1}(t), b_{2}(t), \ldots, b_{m}(t)\right], \Phi$ is a $M N \times M N$ matrix.

In [23], Kilicman and $\mathrm{Al}$ Zhour have given the block pulse operational matrix of the fractional integration $F^{\alpha}$ as follows:

$$
J^{a} B(x) \approx F^{\alpha} B(x),
$$


where

$$
F^{\alpha}=\frac{1}{N M^{\alpha}} \frac{1}{\Gamma(\alpha+2)}\left[\begin{array}{cccccc}
1 & \xi_{1} & \xi_{2} & \xi_{3} & \cdots & \xi_{l-1} \\
0 & 1 & \xi_{1} & \xi_{2} & \cdots & \xi_{l-2} \\
0 & 0 & 1 & \xi_{1} & \cdots & \xi_{l-3} \\
\vdots & \vdots & \vdots & \vdots & \ddots & \vdots \\
0 & 0 & 0 & 0 & \cdots & \xi_{1} \\
0 & 0 & 0 & 0 & \cdots & 1
\end{array}\right]
$$

with $\xi_{k}=(k+1)^{\alpha+1}-2 k^{\alpha+1}+(k-1)^{\alpha+1}$.

Next, we derive the hybrid function operational matrix of the fractional integration. Let

$$
J^{\alpha} H(x) \approx P^{\alpha} H(x)
$$

where matrix $P^{\alpha}$ is called the hybrid function operational matrix of fractional integration.

Using (3.10) and (3.11), we have

$$
J^{\alpha} H(x) \approx J^{\alpha} \Phi B(x)=\Phi J^{\alpha} B(x) \approx \Phi F^{\alpha} B(x) .
$$

From (3.10) and (3.13), we get

$$
P^{\alpha} H(x)=P^{\alpha} \Phi B(x)=\Phi F^{\alpha} B(x) .
$$

Then, the hybrid function operational matrix of fractional integration $P^{\alpha}$ is given by

$$
P^{\alpha}=\Phi F^{\alpha} \Phi^{-1}
$$

Therefore, we have found the operational matrix of fractional integration for hybrid function.

\subsection{The Product Operational of the Hybrid of Block-Pulse and Taylor Polynomials}

The following property of the product of two hybrid function vectors will also be used.

Let

$$
H(x) H^{T}(x) C \cong \widetilde{C} B(x),
$$


see, [22], where $\tilde{C}=\operatorname{diag}\left(\widetilde{C}_{1}, \widetilde{C}_{2}, \ldots, \widetilde{C}_{N}\right)$ is an $M N \times M N$ product operational matrix. And, $\tilde{C}_{i}, i=1,2,3, \ldots, N$ are $M \times M$ matrices given by

$$
\widetilde{C}_{i}=\left(\begin{array}{ccccc}
c_{i 0} & c_{i 1} & c_{i 2} & \cdots & c_{i M-1} \\
0 & c_{i 0} & c_{i 1} & \cdots & c_{i M-2} \\
0 & 0 & c_{i 0} & \cdots & c_{i M-3} \\
\vdots & \vdots & \vdots & \ddots & \vdots \\
0 & 0 & 0 & \cdots & c_{i 0}
\end{array}\right) .
$$

\section{Nonlinear Fredholm Integral Equations}

Considering (1.1), we approximate $D^{\alpha} f(x)$ by the way mentioned in Section 3 as

$$
D^{\alpha} f(x) \approx A^{T} H(x)
$$

For simplicity, we can assume that $\delta_{i}=0$ (in the initial condition). Hence by using (2.5) and (3.13) we have

$$
f(x) \approx A^{T} P^{\alpha} H(x)
$$

Define

$$
\begin{gathered}
C=\left(A^{T} P^{\alpha}\right)^{T} \\
{[f(t)]^{q}=\left[H^{T}(t) C\right]^{q}=\left[C^{T} H(t)\right]^{m}=C^{T} H(t) \cdot H^{T}(t) C\left[H^{T}(t) C\right]^{q-2} .}
\end{gathered}
$$

Applying (3.17) and (4.3) becomes

$$
\begin{aligned}
& {[f(t)]^{q}=A^{T} \tilde{C} H(t)\left[H^{T}(t) C\right]^{q-2}=C^{T} \tilde{C} H(t) \cdot H^{T}(t) C\left[H^{T}(t) C\right]^{q-3},} \\
& {[f(t)]^{q}=C^{T}[\tilde{C}]^{q-1} H(t)=C^{*} H(t) .}
\end{aligned}
$$

With substituting in (1.1) we have

$$
A^{T} H(x)-\lambda \int_{0}^{1} k(x, t) C^{*} H(t) d t=g(x)
$$

We now collocate (4.5) at NM points

$$
x_{n m}=\frac{1}{2 N} \cos \left(\frac{m \pi}{M-1}\right)+\frac{2 n-1}{2 N}, \quad m=0,1,2, \ldots, M-1, n=1,2, \ldots, N
$$


as

$$
A^{T} H\left(x_{n m}\right)-\lambda \int_{0}^{1} k\left(x_{n m}, t\right) C^{*} H(t) d t=g\left(x_{n m}\right) .
$$

We approximate above integral in (4.7) by means of Clenshaw-Curtis rule. Using (4.7), we obtain a system of NM nonlinear equations which can be solved by the Newton's iterative method. By solving this equation we can find the vector $C$.

\section{Error Analysis}

In this section, we discuss the convergence of the hybrid functions method for the nonlinear Fredholm integro-differential equations (1.1).

Suppose we estimate function $f(x) \in L^{2}[0,1]$, using Taylor polynomials of order $M-1$, on the interval $[a, b]$, then using Taylor Residual Theorem, the truncation error is

$$
e(x)=f(x)-\sum_{i=0}^{M-1} \frac{(x-a)^{i}}{i !} f^{(i)}(a)=\frac{(x-a)^{M}}{M !} f^{(M)}(\xi),
$$

see, [24], where $\xi$ lies between $a$ and $x$. Then

$$
\|e(x)\|_{\infty} \leq \frac{(b-a)^{M}}{M !}\left\|f^{(M)}(x)\right\|_{\infty} .
$$

If we use hybrid of block-pulse functions and Taylor series on the interval $[0,1]$, then for $i$ th sub interval $[(i-1) / N, i / N]$, we have

$$
\|e(x)\|_{\infty}=\left\|f(x)-f_{N M}(x)\right\| \leq \frac{1}{N^{M} M !}\left\|f^{(M)}(x)\right\|_{\infty}
$$

see, [25], where the infinity norm is computed on the $i$ th subinterval. It shows that the accuracy improves with increasing the $N$ and $M$.

\section{Numerical Examples}

In this section, we applied the method presented in this paper for solving integral equation of the form (1.1) and solved some examples. All results were computed using MATLAB 7.0.

Example 6.1. Let us first consider fractional nonlinear intego-differential equation:

$$
D_{*}^{\alpha} f(x)-\int_{0}^{1} x t[f(t)]^{2} d t=1-\frac{x}{4}, \quad 0 \leq x<1,0<\alpha \leq 1,
$$

see, [26] with the initial condition $f(0)=0$. 


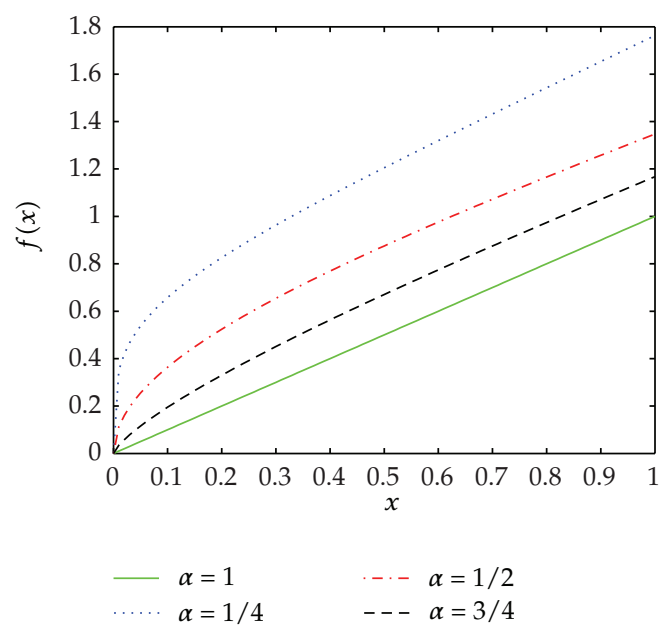

Figure 1: The approximate solution of Example 6.1 for $N=1, M=2$.

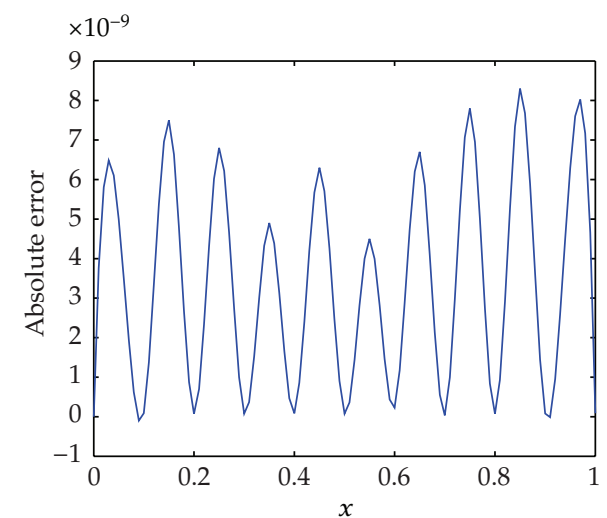

Figure 2: Absolute error of Example 6.3 for $N=10, M=3$.

The numerical results for $M=1, N=2$, and $\alpha=1 / 4,1 / 2,3 / 4$, and 1 are plotted in Figure 1. For $\alpha=1$, the exact solution is given as $f(x)=x$. Note that, as $\alpha$ approaches 1 , the numerical solution converges the analytical solution $f(x)=x$.

Example 6.2. Consider the following equation:

$$
D^{1 / 2} f(x)-\int_{0}^{1} x t[f(t)]^{2} d t=g(x)
$$

where

$$
g(x)= \begin{cases}\frac{1}{\Gamma(1 / 2)}\left(2 x^{1 / 2}-\frac{8}{3} x^{3 / 2}\right)-\frac{11 x}{1920}, & 0 \leq x \leq \frac{1}{2}, \\ \frac{8}{3 \Gamma(1 / 2)} x^{3 / 2}-\frac{21 x}{128}, & \frac{1}{2}<x \leq 1,\end{cases}
$$


Table 1: Absolute error for $\alpha=1 / 2$ and different values of $M, N$ for Example 6.2.

\begin{tabular}{llll}
\hline$x$ & $N=2, M=3$ & $N=2, M=5$ & $N=2, M=7$ \\
\hline 0.1 & $4.6746 e-003$ & $1.5643 e-005$ & $6.0606 e-007$ \\
0.2 & $8.3241 e-003$ & $2.7623 e-005$ & $1.5480 e-006$ \\
0.3 & $7.6758 e-004$ & $3.6753 e-005$ & $4.6970 e-006$ \\
0.4 & $1.3362 e-003$ & $5.0337 e-005$ & $2.2131 e-007$ \\
0.5 & $3.3485 e-003$ & $4.0518 e-005$ & $6.2563 e-007$ \\
0.6 & $1.6673 e-003$ & $6.5000 e-006$ & $2.6769 e-007$ \\
0.7 & $6.0563 e-004$ & $4.2335 e-005$ & $4.7123 e-007$ \\
0.8 & $6.7621 e-004$ & $3.0421 e-005$ & $4.8631 e-006$ \\
0.9 & $1.2309 e-003$ & $3.6676 e-005$ & $2.0707 e-006$ \\
\hline
\end{tabular}

Table 2: Exact and numerical solutions of Example 6.3 for $N=2, M=3$.

\begin{tabular}{lccc}
\hline$x$ & Exact solutions & Numerical solutions & Absolute error \\
\hline 0.2 & 0.04000000 & 0.04023540 & $2.3540 e-004$ \\
0.4 & 0.16000000 & 0.16196810 & $1.9681 e-003$ \\
0.6 & 0.36000000 & 0.36025632 & $2.5632 e-004$ \\
0.8 & 0.64000000 & 0.64035128 & $3.5128 e-004$ \\
1.0 & 1.00000000 & 1.00133210 & $1.3321 e-003$ \\
\hline
\end{tabular}

and with these supplementary conditions $f(0)=0$. The exact solution is

$$
f(x)= \begin{cases}x-x^{2}, & 0 \leq x \leq \frac{1}{2} \\ x^{2}, & \frac{1}{2}<x \leq 1\end{cases}
$$

The absolute error $\left|f(x)-f_{N M}(x)\right|$ for different values of $N$ and $M$ is shown in Table 1 .

Example 6.3. One has

$$
D_{*}^{5 / 3} f(x)-\int_{0}^{1}(x+t)^{2}[f(t)]^{3} d t=g(x), \quad 0 \leq x<1
$$

see, [26] where

$$
g(x)=\frac{6}{\Gamma(1 / 3)} \sqrt[3]{x}-\frac{x^{2}}{7}-\frac{x}{4}-\frac{1}{9}
$$

and with these supplementary conditions $f(0)=f^{\prime}(0)=0$. The exact solution is $f(x)=x^{2}$. Table 2 shows the exact and approximate solution for $N=2, M=3$. Figure 2 illustrates the absolute error $\left|f(x)-f_{N M}(x)\right|$ with $N=10, M=3$. From Figure 2 and Table 2 we can see the numerical solutions are in a good agreement with the exact solution. 


\section{Conclusion}

We have solved the nonlinear Fredholm integral equations of second kind by using hybrid of block-pulse functions and Taylor series. The properties of hybrid of block-pulse functions and Taylor series are used to reduce the equation to the solution of nonlinear algebraic equations. Illustrative examples are given to demonstrate the validity and applicability of the proposed method. The advantages of hybrid functions are that the values of $N$ and $M$ are adjustable as well as being able to yield more accurate numerical solutions. Also hybrid functions have good advantage in dealing with piecewise continuous functions, as are shown.

The method can be extended and applied to the system of nonlinear integral equations, linear and nonlinear integro-differential equations, but some modifications are required.

\section{Acknowledgment}

C. Yang is grateful to the National Natural Science Foundation of China (no. 40806011) for its support.

\section{References}

[1] J. H. He, "Some applications of nonlinear fractional differential equations and their approximations," Bulletin of Science and Technology, vol. 15, pp. 86-90, 1999.

[2] J. A. T. Machado, "Analysis and design of fractional-order digital control systems," Systems Analysis Modelling Simulation, vol. 27, no. 2-3, pp. 107-122, 1997.

[3] I. Hashim, O. Abdulaziz, and S. Momani, "Homotopy analysis method for fractional IVPs," Communications in Nonlinear Science and Numerical Simulation, vol. 14, no. 3, pp. 674-684, 2009.

[4] Ü. Lepik, "Solving fractional integral equations by the Haar wavelet method," Applied Mathematics and Computation, vol. 214, no. 2, pp. 468-478, 2009.

[5] S. Momani and N. Shawagfeh, "Decomposition method for solving fractional Riccati differential equations," Applied Mathematics and Computation, vol. 182, no. 2, pp. 1083-1092, 2006.

[6] S. Momani and M. Aslam Noor, "Numerical methods for fourth-order fractional integro-differential equations," Applied Mathematics and Computation, vol. 182, no. 1, pp. 754-760, 2006.

[7] V. Daftardar-Gejji and H. Jafari, "Solving a multi-order fractional differential equation using Adomian decomposition," Applied Mathematics and Computation, vol. 189, no. 1, pp. 541-548, 2007.

[8] S. Saha Ray, K. S. Chaudhuri, and R. K. Bera, "Analytical approximate solution of nonlinear dynamic system containing fractional derivative by modified decomposition method," Applied Mathematics and Computation, vol. 182, no. 1, pp. 544-552, 2006.

[9] Q. Wang, "Numerical solutions for fractional KdV-Burgers equation by adomian decomposition method," Applied Mathematics and Computation, vol. 182, no. 2, pp. 1048-1055, 2006.

[10] M. Inc, "The approximate and exact solutions of the space- and time-fractional Burgers equations with initial conditions by variational iteration method," Journal of Mathematical Analysis and Applications, vol. 345 , no. 1 , pp. 476-484, 2008.

[11] S. Momani and Z. Odibat, "Analytical approach to linear fractional partial differential equations arising in fluid mechanics," Physics Letters A, vol. 355, no. 4-5, pp. 271-279, 2006.

[12] Z. M. Odibat and S. Momani, "Application of variational iteration method to nonlinear differential equations of fractional order," International Journal of Nonlinear Sciences and Numerical Simulation, vol. 7, no. 1, pp. 27-34, 2006.

[13] S. Momani and Z. Odibat, "Homotopy perturbation method for nonlinear partial differential equations of fractional order," Physics Letters A, vol. 365, no. 5-6, pp. 345-350, 2007.

[14] N. H. Sweilam, M. M. Khader, and R. F. Al-Bar, "Numerical studies for a multi-order fractional differential equation," Physics Letters. A, vol. 371, no. 1-2, pp. 26-33, 2007.

[15] I. Hashim, O. Abdulaziz, and S. Momani, "Homotopy analysis method for fractional IVPs," Communications in Nonlinear Science and Numerical Simulation, vol. 14, no. 3, pp. 674-684, 2009.

[16] E. A. Rawashdeh, "Numerical solution of fractional integro-differential equations by collocation method," Applied Mathematics and Computation, vol. 176, no. 1, pp. 1-6, 2006. 
[17] V. J. Ervin and J. P. Roop, "Variational formulation for the stationary fractional advection dispersion equation," Numerical Methods for Partial Differential Equations, vol. 22, no. 3, pp. 558-576, 2006.

[18] P. Kumar and O. P. Agrawal, "An approximate method for numerical solution of fractional differential equations," Signal Processing, vol. 86, no. 10, pp. 2602-2610, 2006.

[19] F. Liu, V. Anh, and I. Turner, "Numerical solution of the space fractional Fokker-Planck equation," Journal of Computational and Applied Mathematics, vol. 166, no. 1, pp. 209-219, 2004.

[20] S. B. Yuste, "Weighted average finite difference methods for fractional diffusion equations," Journal of Computational Physics, vol. 216, no. 1, pp. 264-274, 2006.

[21] I. Podlubny, Fractional Differential Equations: An Introduction to Fractional Derivati-Ves, Fractional Equations, to Methods of their Solution and Some of their Applications, vol. 198, Academic Press, New York, NY, USA, 1999.

[22] M. Razzaghi, "Optimization of time delay systems by hybrid functions," Optimization and Engineering, vol. 10, no. 3, pp. 363-376, 2009.

[23] A. Kilicman and Z. A. A. Al Zhour, "Kronecker operational matrices for fractional calculus and some applications," Applied Mathematics and Computation, vol. 187, no. 1, pp. 250-265, 2007.

[24] K. Maleknejad and Y. Mahmoudi, "Numerical solution of linear Fredholm integral equation by using hybrid Taylor and block-pulse functions," Applied Mathematics and Computation, vol. 149, no. 3, pp. 799-806, 2004.

[25] Z. H. Jung and W. Schaufelberger, Block Pulse Functions and Their Applications in Control Systems, vol. 179, Springer, Berlin, Germany, 1992.

[26] H. Saeedi, M. Mohseni Moghadam, N. Mollahasani, and G. N. Chuev, "A CAS wavelet method for solving nonlinear Fredholm integro-differential equations of fractional order," Communications in Nonlinear Science and Numerical Simulation, vol. 16, no. 3, pp. 1154-1163, 2011. 


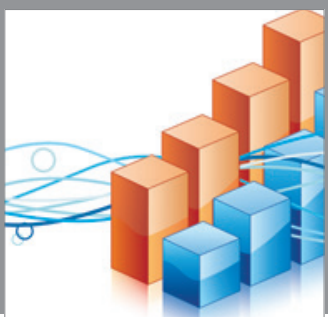

Advances in

Operations Research

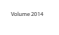

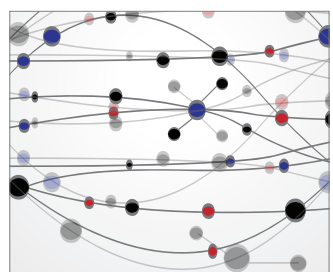

\section{The Scientific} World Journal
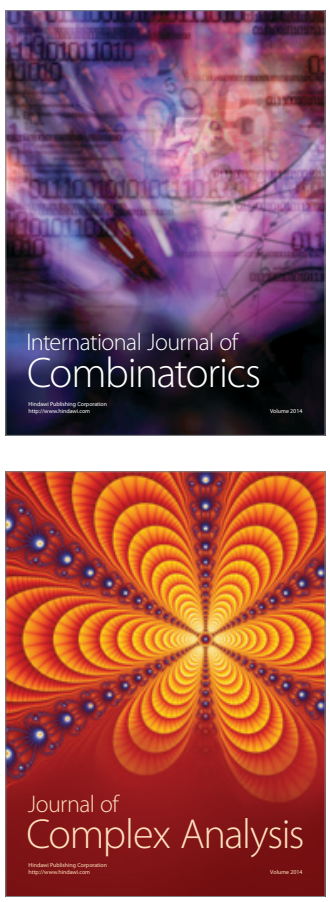

International Journal of

Mathematics and

Mathematical

Sciences
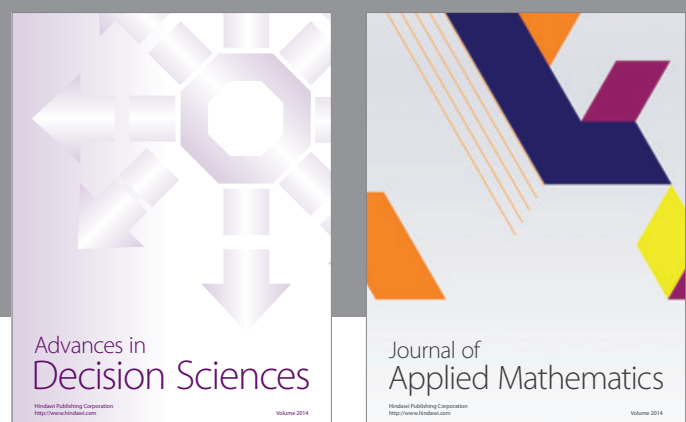

Journal of

Applied Mathematics
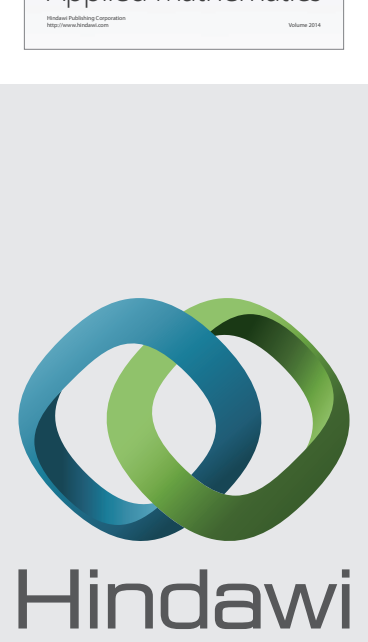

Submit your manuscripts at http://www.hindawi.com
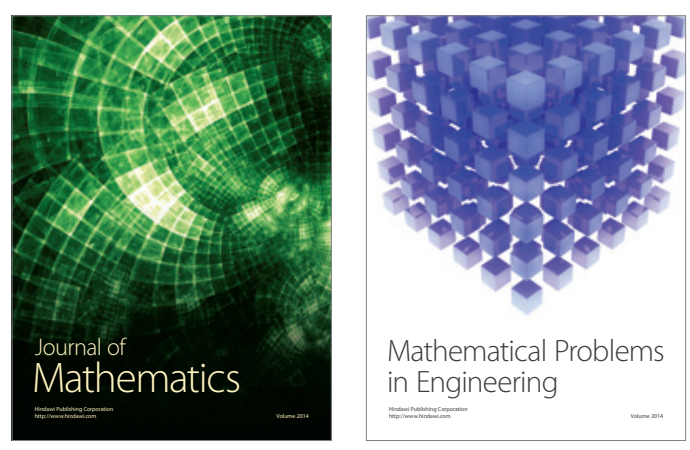

Mathematical Problems in Engineering
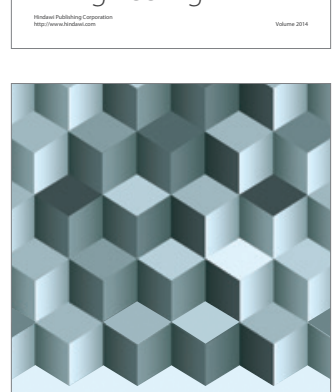

Journal of

Function Spaces
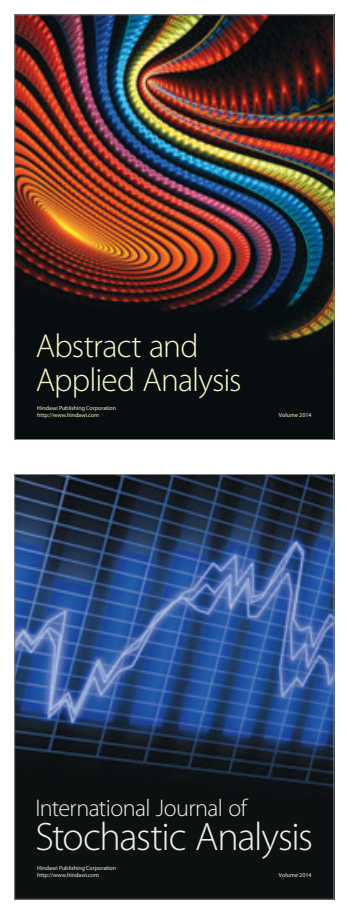

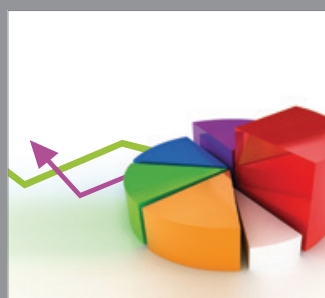

ournal of

Probability and Statistics

Promensencen
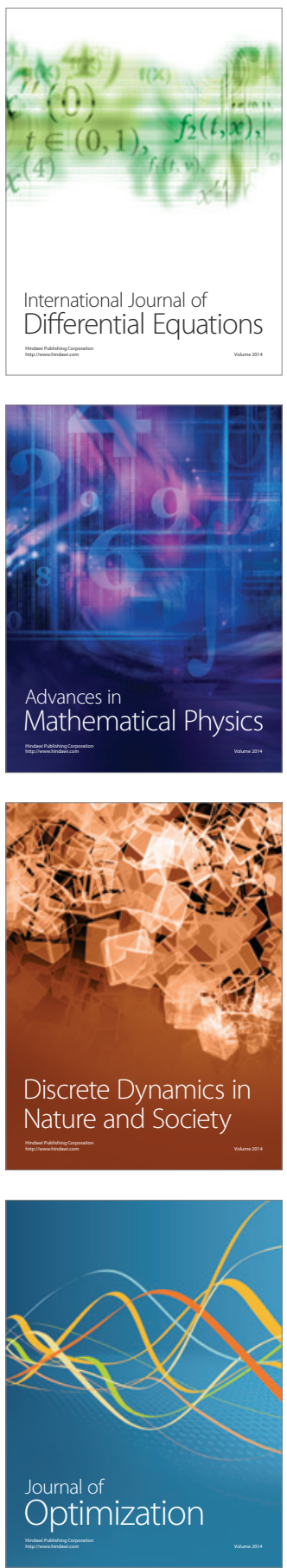